\title{
Preface: Special issue on computing and combinatorics conference and wireless algorithms, systems, and applications conference
}

\author{
Zhipeng Cai ${ }^{1}$ - Chaokun Wang ${ }^{2}$ - Anu Bourgeois ${ }^{1}$
}

Published online: 14 January 2016

(C) Springer Science+Business Media New York 2016

This special issue of the Journal of Combinatorial Optimization consists of 12 selected papers, where half of them were presented at the 20th International Computing and Combinatorics Conference (COCOON 2014), taken place in the city of Atlanta, Georgia, USA, during August 4-6, 2014, and the other half were presented at the 9th International Conference on Wireless Algorithms, Systems, and Applications (WASA 2014), taken place in the city of Harbin, Heilongjiang, China, during June 23-25, 2014.

COCOON 2014 provided a forum for researchers working in the area of theoretical computer science and combinatorics. The technical program of the conference includes 51 contributed papers selected by the program committee from a number of 110 full submissions. 7 extended versions of the articles, selected from these 51 accepted papers, are invited and 6 of them are accepted to this special issue through a regular reviewing process.

WASA 2014 provided a forum for theoreticians, system and application designers, protocol developers and practitioners to discuss and express their views on the current trends, challenges, and state-of-the-art solutions related to various issues in wireless networks. The technical program of the conference includes 40 contributed papers together with 30 invited papers, selected by the program committee from a number

\footnotetext{
Zhipeng Cai

zcai@gsu.edu

Chaokun Wang

chaokun@tsinghua.edu.cn

Anu Bourgeois

abourgeois@cs.gsu.edu

1 Department of Computer Science, Georgia State University, Atlanta, GA 30303, USA

2 The School of Software, Tsinghua University, Beijing 100084, China
} 
of 134 full submissions. 8 extended versions of the articles, selected from these 71 accepted papers, are invited and 6 of them are accepted to this special issue through a regular reviewing process.

We would like to thank the authors for contributing their works, and program committee members and external reviewers for volunteering their time to review the papers. 